\title{
Surface wave attenuation in foothills areas: two innovative approaches
}

Laurent Duval, Martine Ancel, Marc Becquey, Karine Broto*, IFP

\begin{abstract}
Summary
Surface wave attenuation is a difficult problem to address in foothills areas due to the high variability of their typology. Such recorded events can indeed be non linear and even mimic reflection hyperbolas.

We present two innovative approaches that exploit the combination of different criteria so as to discriminate between effective signal and noise. The first one makes use of both apparent velocity and polarisation. Its ability to detect noise is demonstrated on a synthetic multi-component data set inspired from South American foothills. The second one combines apparent velocity and a decomposition on different time and space scales. Improvements over classical FK filtering are shown on a single component real data set from Canadian foothills.
\end{abstract}

\section{Introduction}

The exploration of new petroleum provinces is of strategical importance for the oil industry. Among those poorly explored areas, foothills appear very attractive: $18 \%$ of oil discoveries from 1920 were located in folded and thrust belt areas (source IHS-Energy, 2001). The exploration in foothills areas remains challenging, from acquisition to depth imaging. In particular for the latter, the generally poor $\mathrm{S} / \mathrm{N}$ ratio of the recorded data can seriously hamper the velocity model building phase since interpretable prestack data are required.

Surface waves, which represent most of the coherent noise energy in foothills areas, are very complex to eliminate by classical methods, generally based on apparent velocity considerations such as FK filtering. Due to rugged topography, surface waves do not appear any longer as linear events. Besides, these topography changes as well as heterogeneities in the near surface scatter the propagating surface waves, which can even result in recording hyperbolas if the scattering points are not along the source-receiver line.

We present two new approaches for tackling the problem of surface noise attenuation in foothills areas. The first one is based on a double projection of multi-component data and the second one on a wavelet transform of monocomponent recorded data, both combined with apparent velocity filtering.

\section{Apparent velocity: FK filtering}

Ground roll filtering is generally performed in the industry by FK filtering (Yilmaz, Ö., 2001), which consists in attenuating a range of apparent velocities in the frequency/wavenumber domain. Its principle relies on differences between the velocities of the surface waves and the apparent velocities of the reflections.

In complex areas and in particular in foothills, characterized by strongly dipping layers, rugged topography and heterogeneities in the near surface that act as scattering points, these assumptions generally do not hold any longer. Combining apparent velocity with other criteria provides better results.

\section{Velocity and polarisation: double projection filter}

The double projection filter (Becquey and Rodriguez, 2000) estimates the local polarisation and apparent velocity of the ground roll in the frequency band where it predominates and projects the data onto these vectors. Adding polarisation to velocity as filtering parameters, it requires multicomponent data.

A synthetic 2-component shot was simulated with a $2 \mathrm{D}$ elastic Finite Difference code over a model inspired from a real subsurface encountered in a south american foothills area. This gather was generated taking into account both the topography and a near surface low velocity layer. The shot gather in Figure 1a displays non linear surface waves and scattered noise, related to topography changes and heterogeneities in the near surface.

A first estimation of the ground-roll (Fig. 1c) was subtracted from the data, removing the locally dominant modes of surface waves. Other modes, which were hidden on the original data by more energetic modes, could be estimated by a second application of the filter (Fig. 1d) and finally subtracted to the first residue (Fig. 1b).

Some of the events dipping to the left on the left side of the figure are removed by the filter. Some are not. Examination of the in-line and vertical components shows that a part of these events are surface waves with the two components in quadrature, while another part are dipping reflections with the two components in phase. Both kinds of waves share the same apparent velocity. Taking polarisation into account enables selective attenuation of the surface waves, that would be out of range of FK filtering.

\section{Velocity and scales: directional wavelet frames}

In general, multicomponent data are not available. Ground-roll removal can be performed on single component data by combining apparent velocity and a decomposition into different scales with specific local orientations.

Filtering is traditionally performed through the projection of seismic data onto a basis of functions. Prediction techniques need also to be mentioned, but fall outside the 


\section{Surface wave attenuation in foothills areas}

scope of this work. Let $N_{x}$ and $N_{t}$ be the number of traces and samples per trace. In the case of Fourier transforms, the basis is composed of complex exponentials; it becomes bidimensional in frequency and wave number with the FK transform. Wavelets are widely used for surface wave filtering, since their time-scale variant properties are attractive in dispersive cases. Bases may be arbitrary, but the number of transformed coefficients $c_{n}$ remains the same as the number of signal samples, $N_{x} N_{t}$. Bases are nonredundant. This feature is especially sensitive in the case of filtering: it leads to Gibbs phenomenons with Fourier or wavelet bases. They are also dependent on the basis selection (Miao and Cheadle, 1998).

In several applications, basis limitations are overcome with frames (Duffin and Schaeffer, 1952). In contrast to bases, the frame representation can be made redundant: the signal is decomposed over $N_{f}$ vectors, with $N_{f} \geq N_{x} N_{t}$. As a result, it is much more robust to noise, thus more adequate for denoising. It is also effective in reducing aliasing effects both on noise and data, and shows less sensitivity to the wavelet choice (Duval and Galibert, 2002).

Zhang and Ulrych (Zhang and Ulrych, 2003) propose physical frames representing seismic wavelets convolved with hyperbolas. In the context of foothills, reflections generally do not follow hyperbolas, due to the layer structure. But portions of hyperbolas may be observed. They can be approximated by constant slope segments. As a consequence, we propose the design of local directional frames.

Directional frames are obtained with iterations of $2 \mathrm{D}$ wavelet bases. Such iterations are only useful if noise or ground-roll and signal are not well separated by the current projection. Due to the local behavior of wavelets, local mutes performed in the transformed domain further separate signal from noise where they do not overlap. The resulting separation is thus performed in frequency, direction (or apparent velocity) and space. We refer to (Mallat, 1998) for a comprehensive overview on wavelets and frames.

\section{Application on real data}

Directional frame filtering was applied on the public domain Husky-Talisman line (Central Alberta). Results for one shot gather are reported in Figure 2. This shot clearly exhibits near-surface and topography effects (Fig. 2 , top).

The directional frames are generated by a redundant wavelet packet decomposition based on near symmetric Symlet wavelets (Mallat, 1998, p. 250 sq.). FK filtering (Fig. 2, center) removes the hyperbola branches; applied to $2 \mathrm{D}$ data, it cannot deal with the apices of hyberbolas, generated by the scattered ground-roll. Local scale analysis with wavelet frames (Fig. 2, bottom) better resolves signal from surface waves.

The inherent redundancy in frames also helps reduce others uncoherent noises, yielding clearer reflections.
Wavelets are also well suited to the local unstationarities in the signal.

\section{Conclusions}

In foothills areas, rugged topography, low velocities and heterogeneities in the near surface, strongly dipping layers, unite to reduce the efficiency of FK filtering.

Two innovative approaches, that make use of additional information than the sole apparent velocity, are proposed and estimated on synthetic and real data. Thus, it appears that combining apparent velocity with polarisation for multi-component data or space and time scales with directional frames for mono-component data improves the attenuation of the ground-roll.

The continuity of seismic reflections in prestack gathers being enhanced, a better structural imaging is expected.

\section{Acknowledgements}

We thank Total for authorising to show and use the synthetic data set generated from one of their model inspired from South American foothills, as well as Husky Oil and Talisman Energy for providing the real Central Alberta data set.

\section{References}

Becquey, M., and Rodriguez, S., 2000, Double projection three-component groundroll filter: Annual International Meeting, Soc. of Expl. Geophysicists, Annual International Meeting, 2100-2102.

Duffin, R., and Schaeffer, A., 1952, A class of nonharmonic Fourier series: Trans. Amer. Math. Soc., 72, 341-366

Duval, L. C., and Galibert, P.-Y., 2002, Efficient coherent noise filtering - an application of shift-invariant wavelet denoising: Conference and Technical Exhibition, European Assoc. of Geoscientists and Engineers, C010.

Mallat, S., 1998, A wavelet tour of signal processing: Academic Press.

Miao, X., and Cheadle, S. P., 1998, Noise attenuation with wavelet transforms: Annual International Meeting, Soc. of Expl. Geophysicists, 1072-1075.

Yilmaz, Ö., 2001, Seismic data analysis: Soc. of Expl. Geophysicists.

Zhang, R., and Ulrych, T., 2003, Physical wavelet frame denoising: Geophysics, 68, no. 1, 225-231. 
Surface wave attenuation in foothills areas

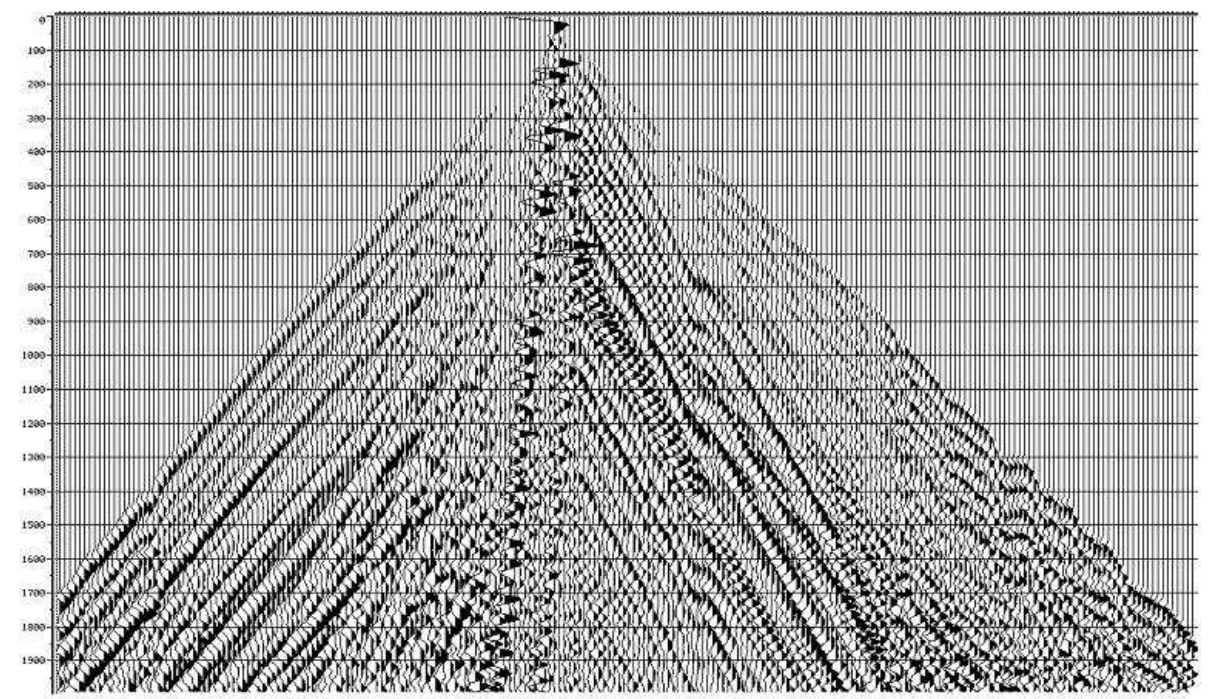

(a)

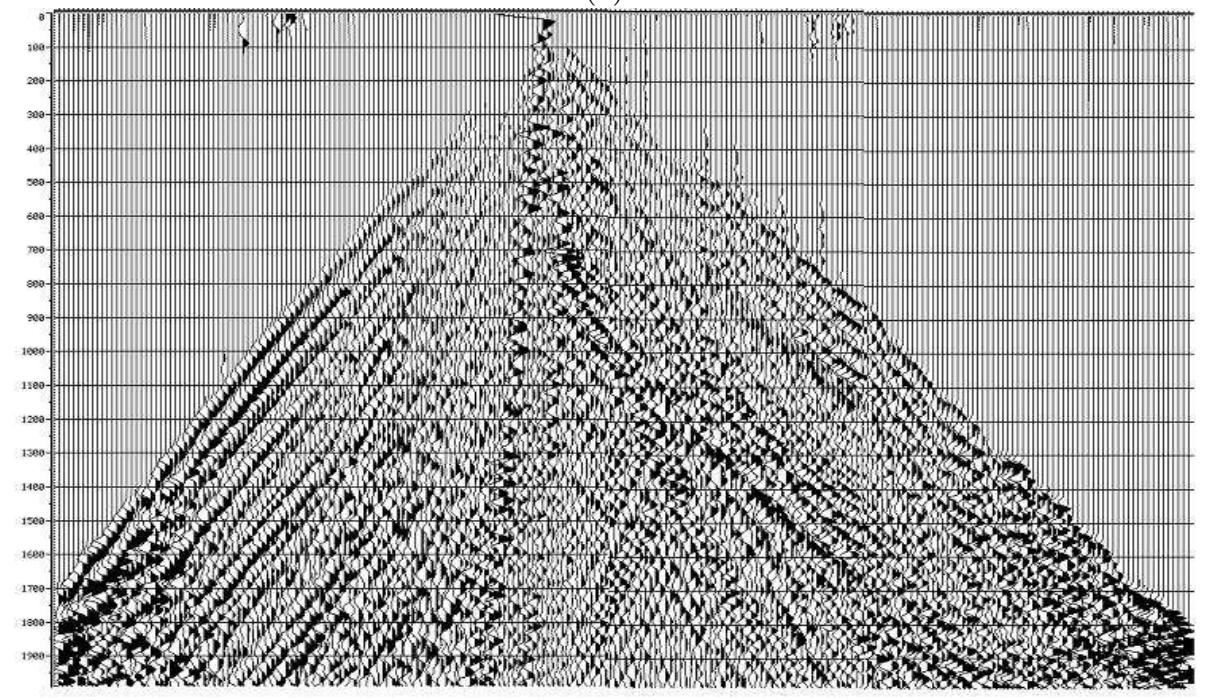

(b)

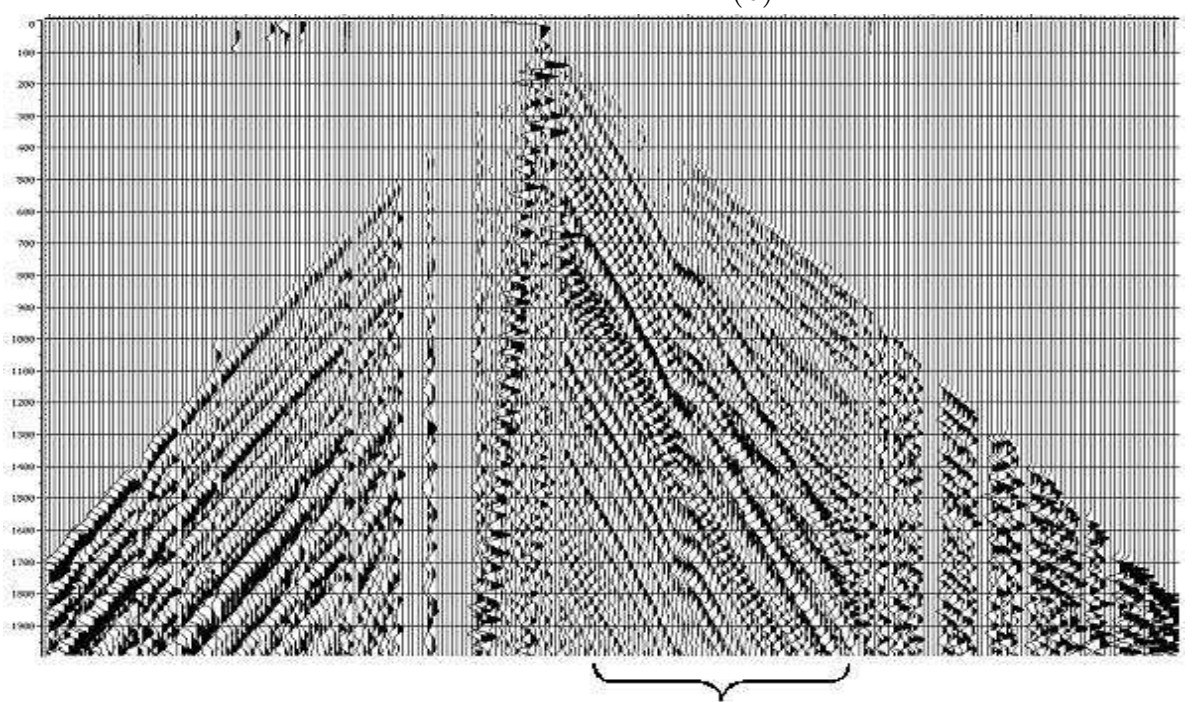

(c)

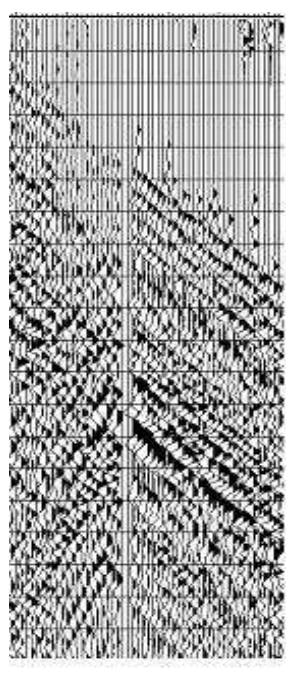

(d)

Fig. 1: Double projection filtering: a) Shot gather modelized with rugged topography and a low velocity layer; b) Filtered data, after substraction of the estimated ground-roll; c) and d) Ground-roll estimation - 1st and 2nd pass . 
Surface wave attenuation in foothills areas
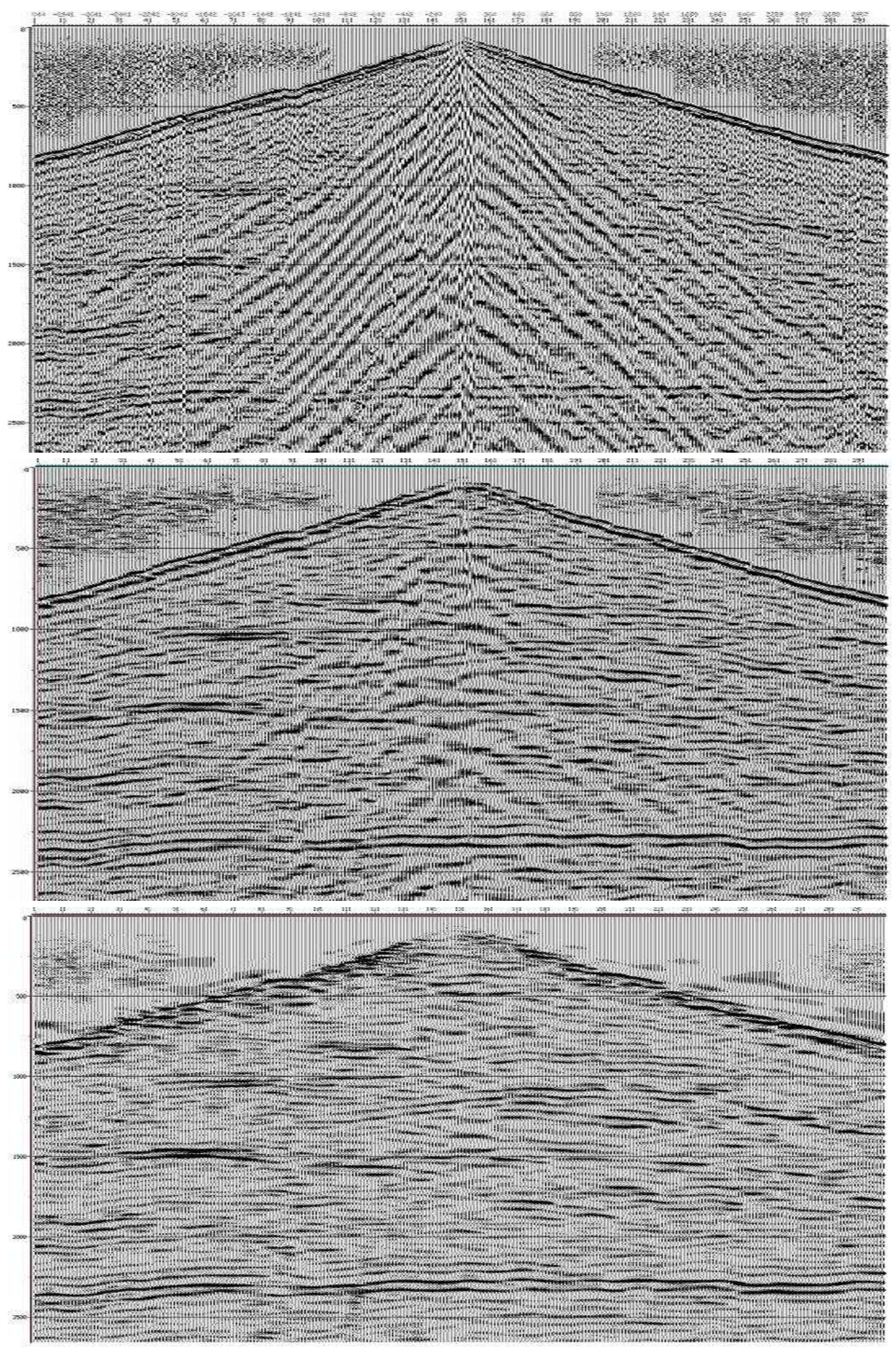

Fig. 2: Groundroll removal on Husky data: (Top) Initial data, (Center) FK filtered data, (Bottom) Data filtered with directional frames. 